THE ASTROPHySiCAL JouRnal SUPPLEMENT SERIES, 90:833-835, 1994 February

(1) 1994. The American Asıronomical Society. All rights reserved. Printed in U.S.A.

\title{
ELECTRODYNAMICS OF DISK-ACCRETING MAGNETIC NEUTRON STARS
}

\author{
M. Coleman Miller, Frederick K. Lamb, ${ }^{1}$ and Russell J. Hamilton \\ Department of Physics, University of Illinois at Urbana-Champaign, 1110 West Green Street, Urbana, IL 61801-3080 \\ Received 1993 February 26; accepted 1993 May 19
}

\begin{abstract}
We have investigated the electrodynamics of magnetic neutron stars accreting from Keplerian disks and the implications for particle acceleration and $\gamma$-ray emission by such systems. We argue that the particle density in the magnetospheres of such stars is larger by orders of magnitude than the Goldreich-Julian density, so that the formation of vacuum gaps is unlikely. We show that even if the star rotates slowly, electromotive forces (EMFs) of order $10^{15} \mathrm{~V}$ are produced by the interaction of plasma in the accretion disk with the magnetic field of the neutron star. The resistance of the disk-magnetosphere-star circuit is small, and hence these EMFs drive very large conduction currents. Such large currents are likely to produce magnetospheric instabilities, such as relativistic double layers and reconnection events, that can accelerate electrons or ions to very high energies.
\end{abstract}

Subject headings: acceleration of particles - accretion, accretion disks - MHD — stars: neutron

\section{INTRODUCTION}

The availability of $\mathrm{MeV}$ to $\mathrm{GeV}$ instruments on board the Compton Observatory and Granat and the improving sensitivity of ground-based $\mathrm{TeV}$ and $\mathrm{PeV}$ detectors have focused attention on whether accreting neutron star systems may produce detectable fluxes of high-energy electromagnetic radiation. Motivated by this question, we have investigated interactions of magnetic neutron stars with Keplerian accretion disks that may lead to the production of $\gamma$-rays. Here we present a summary of this work, based on a more detailed account to be published elsewhere (Lamb, Hamilton, \& Miller 1994; see also Lamb, Hamilton, \& Miller 1992; Miller, Lamb, \& Hamilton 1993).

\section{DISK-STAR INTERACTION}

Consider a system in which a neutron star with a strong dipolar magnetic field is accreting plasma from a Keplerian disk. For simplicity, assume that the neutron star magnetic and rotation axes are aligned and that the star is slowly rotating. (We expect the qualitative features of the system to be as described below, even if the axes are not aligned.) Assume also that the disk is geometrically thin and situated in the rotational equatorial plane of the neutron star. In analyzing this system, we use cylindrical coordinates $(\varpi, \phi, z)$ centered on the star. Our analysis focuses on the general features of the system rather than any particular model.

Plasma near the neutron star is highly ionized and has a high electrical conductivity. We therefore expect an electric field $\boldsymbol{E} \approx-\boldsymbol{v} \times \boldsymbol{B} / \boldsymbol{c}$ in this region, which causes the plasma to corotate with the star. The magnitude of the charge density near the star is then (see Goldreich \& Julian 1969)

$$
\begin{aligned}
\left|n_{+}-n_{-}\right| \approx n_{\mathrm{GJ}} \approx\left|\Omega_{s} \cdot \boldsymbol{B}\right| / 2 \pi e c & \\
& =7 \times 10^{4} \mu_{30} r_{8}^{-3} P_{s}^{-1} \mathrm{~cm}^{-3}
\end{aligned}
$$

\footnotetext{
${ }^{1}$ Also Department of Astronomy.
}

where $\Omega_{s}$ is the stellar angular frequency, $\mu_{30}$ is the stellar magnetic moment in units of $10^{30} \mathrm{G} \mathrm{cm}^{3}, r_{8}$ is the radius in units of $10^{8} \mathrm{~cm}$, and $P_{s}$ is the stellar rotation period in seconds. We also expect the disk to have a magnetic field that causes the highly conducting plasma near the disk to rotate with an angular velocity close to that of the disk plasma to which it is connected by the magnetic field. The magnitude of the charge density in this region is given approximately by equation (1), with $P_{s}$ replaced by the local rotation period.

We expect the charge density near the neutron star and the inner disk to be much smaller than the particle density there, for two reasons. First, the electrical potential energy difference between the inner disk and the star is $\sim 10^{15} \mathrm{eV}$ ( see below), whereas the gravitational potential energy confining protons to the disk is only $\sim 10^{6} \mathrm{eV}$. Hence, even small fluctuations in the electric field near the disk caused by irregularities in the accretion flow can readily pull protons as well as electrons out of the disk into the magnetosphere. Second, about $10^{40}-10^{42}$ particles per second flow from the disk to the neutron star. The differing rotation rates of the star and disk plasma twist the magnetic field in this region, creating MHD instabilities that inject plasma into the magnetosphere above the inner disk ( see Ghosh \& Lamb 1991). Even if only a fraction $\sim 10^{-10}$ of the accreting plasma is injected with a velocity comparable to the local Keplerian velocity, the particle density in the magnetosphere greatly exceeds $n_{\mathrm{GJ}}$. Indeed, X-ray observations of Her X-1 (Becker et al. 1977; Bai 1980) indicate that the particle density above the inner disk is $\gtrsim 10^{11} \mathrm{~cm}^{-3}$, far greater than $n_{\mathrm{GJ}}$. Formation of a vacuum gap in the magnetosphere requires complete charge separation (see Cheng \& Ruderman 1991 ) and therefore seems unlikely. Our treatment of the interaction between the disk and stellar magnetic field is based on the theory of disk-accreting neutron stars developed over the last 15 years, which assumes that the particle density in the magnetosphere is large enough to support substantial conduction currents (see Ghosh \& Lamb 1991).

The magnetic field of the star can interact with plasma in the disk in a variety of ways. The processes that transport angular momentum in the disk-such as turbulence and magnetic 
coupling - by their nature allow magnetic flux from the star to couple to the inner part of the disk. The differential rotation of the disk plasma and star generates an EMF that drives fieldaligned currents within the magnetosphere. These currents create an azimuthal magnetic field component. The process can be thought of as twisting of the magnetic field due to the differing rotation rates of the disk plasma and star. The resulting magnetic stress on the disk extracts angular momentum from the disk plasma. The Keplerian flow ends at the radius $\varpi_{0}$ where the magnetic stress becomes so large that it extracts the angular momentum of the disk plasma in a radial distance much less than $\varpi$ (see Ghosh \& Lamb 1991). Inside $\varpi_{0}$, the angular velocity $\Omega_{d}$ of the disk plasma falls rapidly from the Keplerian value $\Omega_{K}$, reaching the angular velocity of the star at $\varpi_{\mathrm{co}}$.

\section{SYSTEM ELECTRODYNAMICS}

Consider now the electrodynamics of this system. We first calculate the EMF

$$
\mathscr{E} \equiv \oint_{C}\left(\boldsymbol{E}+\frac{1}{c} v \times \boldsymbol{B}\right) \cdot d \boldsymbol{l}
$$

around the closed path $C$ denoted by the sequence of points $1-2-3 \cdots 8-1$ shown in Figure 1 . As a first approximation, we assume that the electrical resistance of the matter along this path is negligible.

Noting that $v \times B \cdot d l=0$ wherever $C$ follows the poloidal

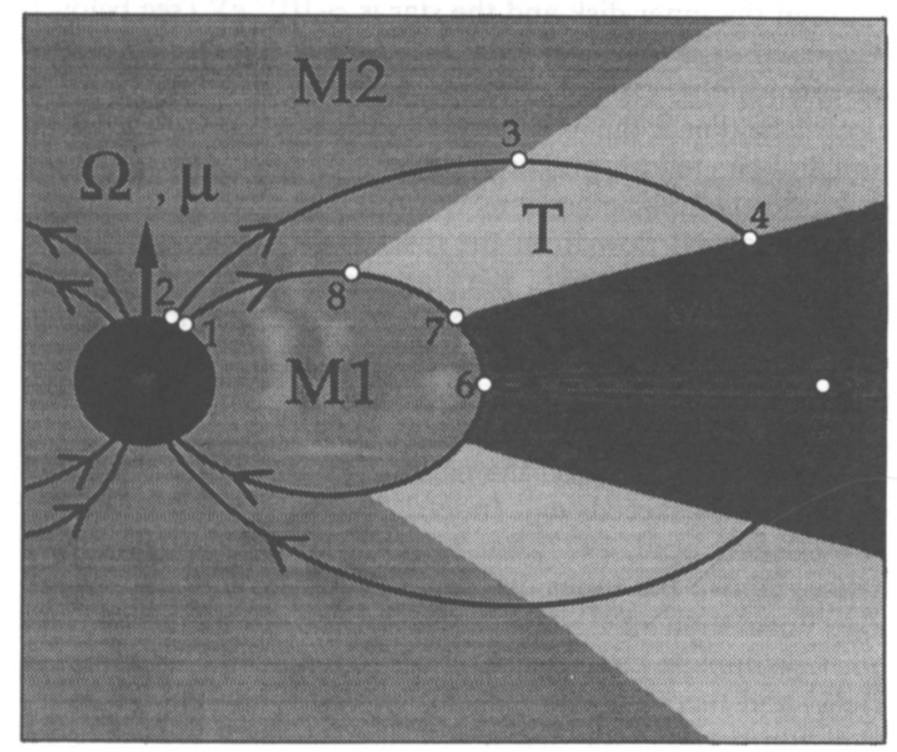

FIG. 1.-Schematic diagram of the system analyzed in the text. Plasma on the magnetic field line that connects the inner edge of the disk to the stellar surface (points 6-7-8-1) corotates with the star, as does plasma in regions $\mathrm{M} 1$ and $\mathrm{M} 2$. Plasma in region $\mathrm{M} 3$ orbits the star with the angular velocity of the adjacent disk plasma. We expect velocities in the transition region $T$ to be intermediate between the angular velocity of the star and that of the adjacent disk plasma. The size and shape of region $T$ depends on the particular model.

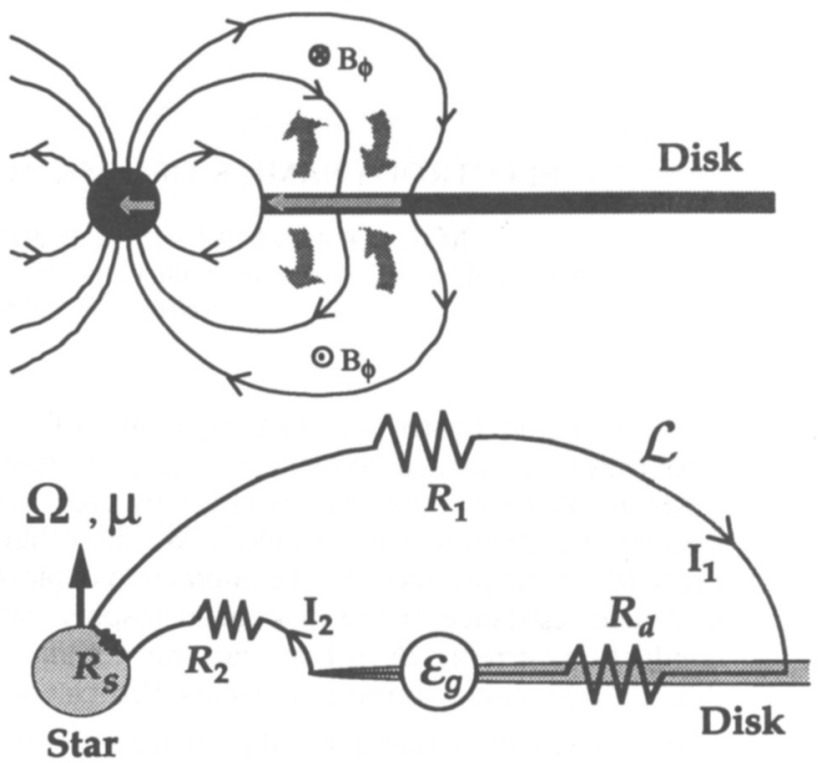

FIG. 2.-(Top) Orbiting motion of the disk plasma generates an EMF that drives cross-field currents (light shaded arrows) within the disk and the neutron star and field-aligned currents (shaded arrows) within the magnetosphere. (Bottom) Equivalent circuit, showing the generated EMF $\mathscr{E}_{g}$, the circuit inductance $\mathscr{L}$, and the resistances $\mathscr{R}_{s}$ and $\mathscr{R}_{d}$ of the star and disk. Also shown are the resistances $\mathscr{R}_{1}$ and $\mathscr{R}_{2}$ and the currents $I_{1}$ and $I_{2}$ in the two magnetospheric legs of the circuit.

field, we find

$$
\begin{aligned}
\mathscr{E}= & -\frac{1}{c} \int_{A} \frac{\partial B_{\phi}}{\partial t} d A \\
& +\frac{1}{c} \int_{1}^{2}\left(\Omega_{s} \times \boldsymbol{r}\right) \times \boldsymbol{B} \cdot d \boldsymbol{l}+\frac{1}{c} \int_{s}^{6}\left(\Omega_{d} \times \boldsymbol{r}\right) \times \boldsymbol{B} \cdot d \boldsymbol{l} .
\end{aligned}
$$

The first term on the right of this equation comes from the line integral of the electric field around $C$ and describes the backEMF produced by the inductance of the circuit. The second and third terms come from the line integral of $v \times B / c$. We call the sum of the latter two terms the "generated EMF" and denote it $\mathscr{E}_{g}$.

If the neutron star is slowly rotating, the third term in equation (3) dominates the second and

$$
\mathscr{E}_{g} \sim \varpi_{0}^{2} \Omega_{\mathrm{K}}\left(\varpi_{0}\right) B_{p}\left(\varpi_{0}\right) / c \sim 10^{15} \varpi_{8}^{-5 / 2} \mu_{30}\left(M / M_{\odot}\right)^{1 / 2} \mathrm{~V},
$$

where $\Omega_{\mathrm{K}}\left(\varpi_{0}\right)$ and $B_{p}\left(\varpi_{0}\right)$ are the Keplerian angular velocity and the poloidal magnetic field strength at the inner edge of the Keplerian flow. Thus, even if the neutron star is slowly rotating, its interaction with the disk generates very large EMFs.

\section{CURRENTS AND INSTABILITIES}

The large EMFs in the star-magnetosphere-disk circuit drive large electrical currents between the disk and the star. Although the details of the current distribution depend on the model, all produce current patterns qualitatively similar to the pattern shown in the top panel of Figure 2. The bottom panel shows an equivalent circuit that includes the electrical resistance of the disk, star, and magnetosphere. The time develop- 
ment of the current in this circuit is described by the equation

$$
\mathscr{E}_{g}=\mathscr{L} \frac{d I}{d t}+I \mathscr{R}
$$

The term on the left describes the EMF generated by the diskstar interaction, while the first term on the right describes the effect of the circuit inductance $\mathscr{L} \sim r^{2} / c$. The second term on the right describes the effect of the total resistance $\mathscr{R}$ in the circuit.

Equation (4) shows that an initially small current grows linearly with time, corresponding to the linear increase of the azimuthal magnetic field $B_{\phi}$ as the field is twisted by the differential rotation of the disk and star. If no other processes intervened, the growth would slow after a time $\sim \mathscr{L} / \mathscr{R}$, and the current would asymptotically approach the value $\mathscr{E}_{g} / \mathscr{R}$. Our calculations of the resistance $\mathscr{R}$ of the circuit show that it is very small and hence that the asymptotic current would be very large, producing a magnetic pitch $\gamma_{\phi} \equiv B_{\phi} / B_{p} \sim 10^{10}$. We argue (Hamilton, Lamb, \& Miller 1993a,b) that long before the current reaches this value, microscopic and/or MHD instabilities will intervene, interrupting the growth of the current and the twisting of the magnetic field, and accelerating particles to very high energies. Thus, instead of "shorting out" the EMF and thereby preventing acceleration of particles, as some authors have argued (see, e.g., Wang 1986; Katz \& Smith 1988; Mitra 1991), the low internal resistance of the disk-star generator circuit allows these currents to become large enough to trigger processes that accelerate particles.

This research was supported in part by NSF grant PHY 9100283 and NASA grant NAGW 1583 at the University of Illinois.
Bai, T. 1980, ApJ, 239, 328

Becker, R. H., et al. 1977, ApJ, 214, 879

Cheng, K. S., \& Ruderman, M. 1991, ApJ, 373, 187

Ghosh, P., \& Lamb, F. K. 1991, in Neutron Stars: Theory and Observa-

tion, ed. J. Ventura \& D. Pines (Dordrecht: Kluwer), 363

Goldreich, P., \& Julian, W. H. 1969, ApJ, 157, 869

Hamilton, R. J., Lamb, F. K., \& Miller, M. C. 1993a, ApJS, 89, 00 $.1993 \mathrm{~b}$, in preparation

Katz, J. I., \& Smith, I. A. 1988, ApJ, 326, 733

\section{REFERENCES}

Lamb, F. K., Hamilton, R. J., \& Miller, M. C. 1992, in Isolated Pulsars, ed. K. Van Riper, R. Epstein, \& C. Ho (Cambridge: Cambridge Univ. Press), in press .1994 , in preparation

Miller, M. C., Lamb, F. K., \& Hamilton, R. J. 1993, in Proc. Compton Observatory Symposium, ed. N. Gehrels (New York: AIP), 433

Mitra, A. 1991, ApJ, 370, 345

Wang, Y. M. 1986, Ap\&SS, 121, 193 